DEUTSCHE AKADEMIE DER WISSENSCHAFTEN ZU BERLIN VORTRÄGE UND SCHRIFTEN HEFंT 26

\title{
ÜBER
}

\section{DAS NATURRECHT}

von Prof. Dr. Heinrich Mitteis 
Erschienen im Akademie-Verlag GmbH., Berlin NW 7, Schiffbauerdamm 19 Veröffentlicht unter der Lizenznummer $156 \mathrm{der}$ SMA in Deutschland Gedruckt in der Buchdruckerei Oswald Schmidt GmbH., Leipzig M 118 Bestell- und Verlagsnummer 2003/26 